\title{
Summary of Terra and Aqua MODIS Long-term Performance
}

\author{
Xiaoxiong (Jack) Xiong ${ }^{1}$, Brian N. Wenny ${ }^{2}$, Amit Angal ${ }^{3}$, William Barnes ${ }^{4}$, and Vincent Salomonson ${ }^{5}$ \\ ${ }^{1}$ Code 614.4, Sciences and Exploration Directorate, NASA/GSFC, Greenbelt, MD 20771, USA, \\ ${ }^{2}$ Sigma Space Corp., 4801 Forbes Boulevard, Lanham, MD 20706, USA, \\ ${ }^{3}$ Sciences and System Applications Inc., 10210 Greenbelt Road, Lanham, MD 20706, USA, \\ ${ }^{4}$ University of Maryland, Baltimore County, 1000 Hilltop Circle, Baltimore, MD 21250, USA, \\ ${ }^{5}$ University of Utah, Salt Lake City, UT 84112, USA
}

\begin{abstract}
Since launch in December 1999, the MODIS ProtoFlight Model (PFM) onboard the Terra spacecraft has successfully operated for more than 11 years. Its Flight Model (FM) onboard the Aqua spacecraft, launched in May 2002, has also successfully operated for over 9 years. MODIS observations are made in 36 spectral bands at three nadir spatial resolutions and are calibrated and characterized regularly by a set of on-board calibrators (OBC). Nearly 40 science products, supporting a variety of land, ocean, and atmospheric applications, are continuously derived from the calibrated reflectances and radiances of each MODIS instrument and widely distributed to the world-wide user community. Following an overview of MODIS instrument operation and calibration activities, this paper provides a summary of both Terra and Aqua MODIS long-term performance. Special considerations that are critical to maintaining MODIS data quality and beneficial for future missions are also discussed.
\end{abstract}

Index Terms - MODIS, Terra, Aqua, Calibration

\section{INTRODUCTION}

MODIS is a key instrument for NASA's Earth Observing System (EOS). Since launch, its ProtoFlight Model (PFM) has successfully operated onboard the Terra spacecraft for more than 11 years and its Flight Model (FM) onboard the Aqua spacecraft has also successfully operated for more than 9 years. MODIS observations are made in 36 spectral bands, including 20 reflective solar bands (RSB) and 16 thermal emissive bands (TEB), and at 3 spatial resolutions at nadir, $0.25 \mathrm{~km}$ for bands 1-2, $0.5 \mathrm{~km}$ for bands 3-7, and 1.0 $\mathrm{km}$ for the remaining spectral bands. Since launch, both Terra and Aqua MODIS have produced an unprecedented amount of data products and significantly contributed to Earth remote sensing research activities and applications [1]. In addition to design improvements made over its heritage sensors in terms of its spectral range, spatial resolutions, and data coverage, MODIS was developed with very stringent calibration requirements to be met throughout its entire mission. As a result, the MODIS instrument was built with a complete set of on-board calibrators (OBC), including a solar diffuser (SD), a solar diffuser stability monitor (SDSM), a blackbody (BB), and a spectroradiometric calibration assembly (SRCA) [2].

Both Terra and Aqua MODIS went through extensive pre-launch calibration and characterization activities. Their OBC were also fully characterized by the instrument vendor using well calibrated ground testing equipment. During its on-orbit operation, the on-board BB is constantly controlled at a nominal temperature while the SD/SDSM and SRCA are operated on a regular basis. MODIS OBC are used to track changes in sensor radiometric, spectral, and spatial properties and to support MODIS level 1B (L1B) look-uptable (LUT) updates and algorithm improvements [2-5]. This paper provides an overview of both Terra and Aqua MODIS on-orbit operation and calibration activities and an update to their long-term performance. Although having operated beyond their specified design life of 6 years, onorbit performance shows that both Terra and Aqua MODIS instruments as well as their on-board calibrators are still able to provide satisfactory functions and allow high quality data products to be continuously generated. In general, Aqua MODIS has shown better performance than its predecessor, Terra MODIS. Also discussed in this paper are special issues that are critical to the L1B calibration quality and lessons learned that could benefit future sensor development and operation.

\section{OPERATION AND CALIBRATION}

MODIS on-orbit operation and calibration activities are planned and implemented by the MODIS Characterization Support Team (MCST) at NASA/GSFC. Since launch, Terra MODIS has operated using A-side configuration (launch to October 2000), B-side configuration (October 2000 to June 2001), then A-side configuration (July 2001 to September 2002), and finally a configuration with A-side 
electronics and the B-side formatter (September 2002 to present). In addition to a few spacecraft and sensor anomalies and events during the early part of the mission, Terra MODIS has experienced an SD door operational anomaly in May 2003. As a result, the SD door was purposely fixed at the open position with its screen in place starting from July 2003. On the other hand, the Aqua MODIS operation has been very stable. Except for being in the safe mode for a few very short periods due to spacecraft related anomalies at mission beginning, Aqua MODIS has operated continuously using the B-side configuration. The use of B-side electronic configuration for Aqua MODIS onorbit operation was based on both A-side and B-side performance determined from extensive pre-launch calibration and characterization.

MODIS on-orbit calibration activities include regular SD and SDSM calibration events and near-monthly lunar observations for the RSB. In an effort to establish on-orbit calibration scale for each RSB detector and track changes in its response over time, the SD/SDSM operations were scheduled more frequently at the beginning of both Terra and Aqua missions. Normally, the BB temperatures are constantly controlled for the scan-by-scan TEB calibration. Periodic BB warm-up and cool-down (WUCD) operations with $\mathrm{BB}$ temperatures varying between $272 \mathrm{~K}$ and $315 \mathrm{~K}$ are also carried out to support the TEB on-orbit calibration. The SRCA is operated monthly in the radiometric mode but less frequently for the spectral and spatial modes. As shown in Table 1, from launch to present (May 04, 2011), Terra MODIS has scheduled and executed more than 320 SD/SDSM operations, 100 scheduled lunar observations, 75 BB WUCD cycles, and 240 SRCA calibration events (radiometric, spectral, and spatial combined). As expected, the corresponding numbers of Aqua MODIS OBC operations are smaller than that of Terra MODIS. In addition to its OBC and lunar observations, several special operational activities, including spacecraft maneuvers, have been carried out to improve MODIS on-orbit calibration and data quality [3-5].

Table 1 Summary of MODIS on-orbit calibration activities (as of May 04, 2011).

\begin{tabular}{|c|c|c|c|c|c|c|}
\hline & $\begin{array}{c}\text { SD / } \\
\text { SDSM }\end{array}$ & MOON & $\begin{array}{c}\text { BB } \\
\text { WUCD }\end{array}$ & $\begin{array}{c}\text { SRCA } \\
\text { RAD }\end{array}$ & $\begin{array}{c}\text { SRCA } \\
\text { SPET }\end{array}$ & $\begin{array}{c}\text { SRCA } \\
\text { SPAT }\end{array}$ \\
\hline Terra & 322 & 102 & 76 & 133 & 48 & 63 \\
\hline Aqua & 212 & 81 & 39 & 105 & 27 & 45 \\
\hline
\end{tabular}

\section{ON-ORBIT PERFORMANCE}

Terra and Aqua MODIS instrument temperatures are illustrated in Figures 1 and 2 on a weekly average basis over their respective missions. The temperatures during the periods with special calibration activities and events are excluded from the averages. At the beginning of its mission, Terra MODIS operation was carried out using different configurations. Since September 2002, it has been operated using a configuration combing A-side electronics with the B-side formatter. The use of different electronic configurations for Terra MODIS has led to small noticeable discontinuities in its instrument temperatures. Aqua MODIS, however, has been using the same B-side configuration over its entire mission. The long-term changes in instrument temperatures are generally very small, less than $3.5 \mathrm{~K}$ for Terra MODIS over 11 years and less than 2.0 $\mathrm{K}$ for Aqua MODIS over 9 years. The seasonal oscillations are expected and the peak-to-peak variations are typically within $2.0 \mathrm{~K}$. Figures 1 and 2 demonstrate that the spacecraft and MODIS instrument operation environment and conditions have been very stable over their entire missions. The temperatures of the MODIS scan cavity, the scan mirror, and the warm focal plane assemblies (FPA) for the VIS and NIR spectral bands all show a nearly identical trend to the instrument temperatures.

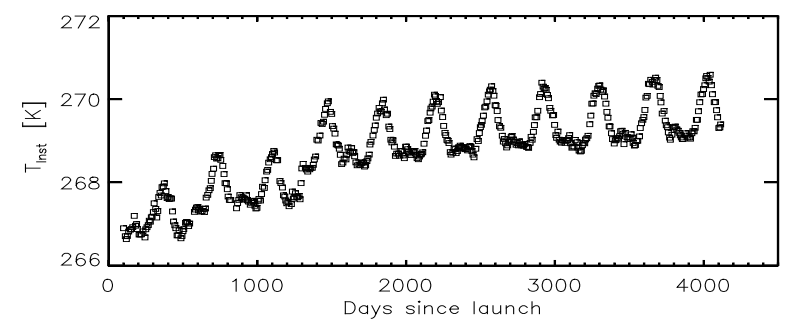

Figure 1 Terra MODIS instrument temperatures

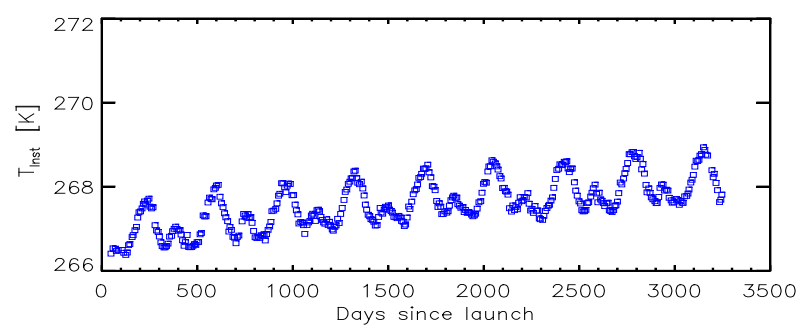

Figure 2 Aqua MODIS instrument temperatures

The SWIR and MWIR spectral bands are located on the same FPA, called the SMIR FPA. It is nominally controlled at 83K together with another cold FPA, which hosts all the LWIR spectral bands. Figures 3 and 4 illustrate Terra and Aqua MODIS LWIR FPA temperatures, which are also on a weekly average basis. Ignoring changes caused by switching between different operational configurations, the cold plane assembly (FPA) temperatures are much better controlled in Terra MODIS than Aqua MODIS. The gradual small increase and noticeable fluctuations of the Aqua cold FPA temperatures in recent years are primarily due to the loss of its radiative cooler margin. Approximately starting from 2007, Aqua MODIS cold FPA temperature has increased about $0.15 \mathrm{~K}$. In addition to seasonal variations and long-term increase shown in Figure 4, there exist similar orbit-to-orbit and daily variations in the Aqua MODIS cold FPA temperatures. 


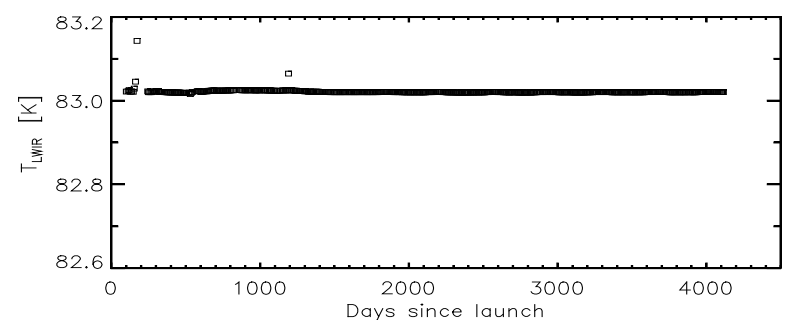

Figure 3 Terra MODIS LWIR FPA temperatures

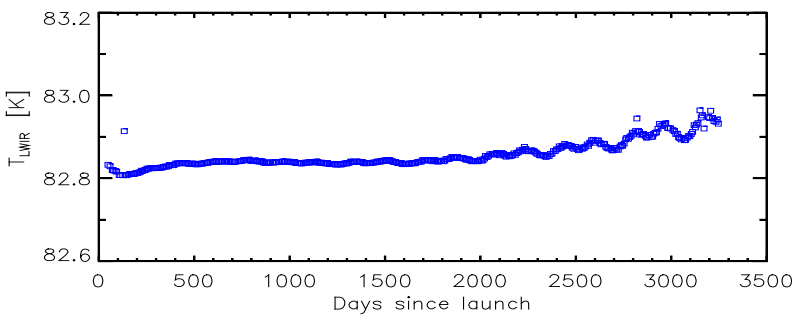

Figure 4 Aqua MODIS LWIR FPA temperatures

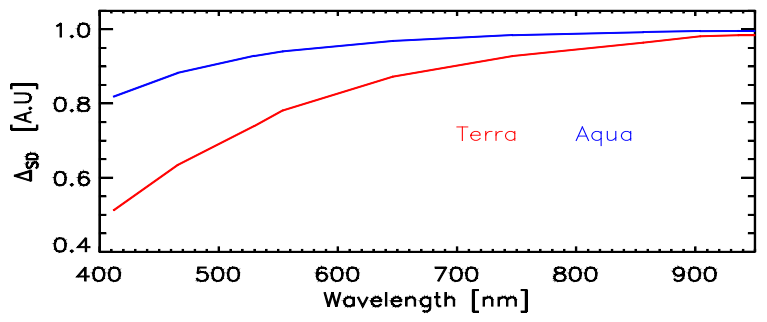

Figure 5 MODIS SD on-orbit degradation (launch to present)

The SDSM is operated during each scheduled SD calibration. It is used to track on-orbit changes in SD bidirectional reflectance factor (BRF). Figure 5 shows SD degradation for both Terra and Aqua MODIS. The SD calibration system was designed with an SD door. Normally it is closed except during regularly scheduled SD and SDSM observations. When both Terra and Aqua MODIS SD and SDSM were operated at the same frequency (or under the same condition) at the beginning of their missions, their SD degradation rates were nearly identical. Following an SD door operational anomaly that led to the decision to fix the SD door at the "Open" position in the middle of 2003, the Terra MODIS SD on-orbit degradation has become much faster than Aqua MODIS. As shown in Figure 5, changes in SD BRF are approximately $49 \%$ at $0.41 \mu \mathrm{m}, 29 \%$ at 0.53 $\mu \mathrm{m}$, and $15 \%$ at $0.65 \mu \mathrm{m}$ for Terra MODIS over 11 years, compared to the changes of $18 \%, 8 \%$, and $4 \%$ at the corresponding wavelengths for Aqua MODIS over 9 years. Clearly, the SD degradation rate is strongly wavelength dependent for both Terra and Aqua MODIS with larger changes observed at shorter wavelengths.

MODIS TEB calibration uses a quadratic algorithm with its linear gains computed from $\mathrm{BB}$ observations on a scan-by-scan basis. Terra MODIS BB is normally controlled at $290 \mathrm{~K}$ and Aqua MODIS at $285 \mathrm{~K}$. Their weekly averaged BB temperatures are presented in Figures 6 and 7.
BB temperatures during periodic WUCD events and other special calibration events are not included in the weekly averages. It is clear that Aqua MODIS BB operation has been extremely stable and there is essentially no long-term drift in the BB temperatures. For Terra MODIS, however, there exist a few small but expected changes due to the use of different instrument operational configurations. In addition, there are small seasonal variations of up to $\pm 0.1 \mathrm{~K}$ for Terra MODIS BB temperatures that are closely matched to seasonal oscillations of instrument temperatures and a small long-term drift of approximately $0.03 \mathrm{~K}$.

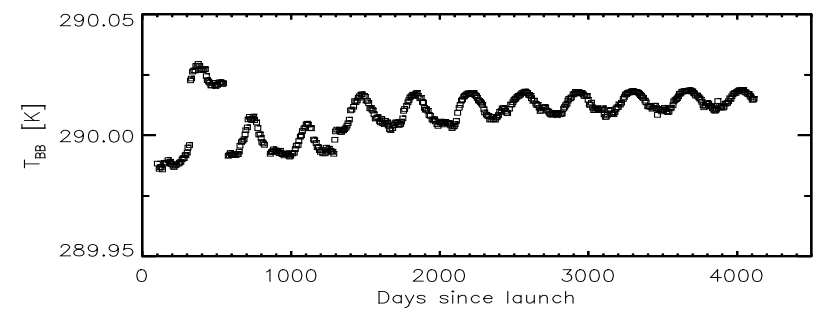

Figure 6 Terra MODIS on-board BB temperatures

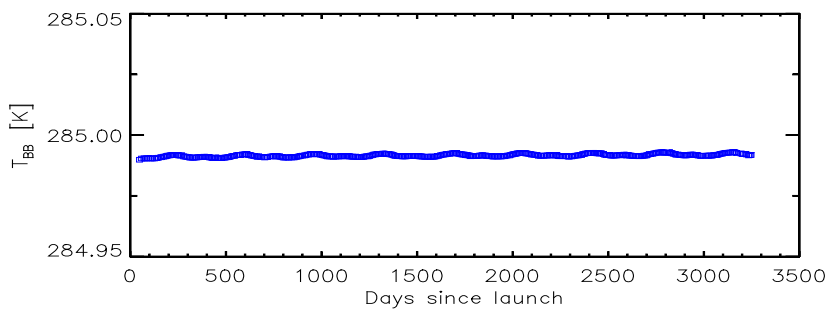

Figure 7 Aqua MODIS on-board BB temperatures

In general, larger changes in detector responses (or gains) are observed at shorter wavelengths for the RSB, which also have noticeable mirror side and scan angle dependencies. Figure 8 shows on-orbit changes in mirror side dependent gains derived from $\mathrm{SD}$ and lunar observations for Terra MODIS band 8 at $0.41 \mu \mathrm{m}$. The SD and lunar observations are made at 2 different angles of incidence (AOI) to the scan mirror. For Aqua MODIS, as shown in Figure 9, the mirror side differences are relatively small. Changes, including mirror side differences, are much smaller for the spectral bands of longer wavelengths, such as the SWIR bands and TEB. In addition to changes in detector long-term responses, small short-term variations, less than $\pm 1.0 \%$, exist in recent Aqua MODIS TEB LWIR detector responses, which are caused by variations in the cold FPA temperatures (Figure 4). The short-term variations, however, have little impact on data quality as MODIS TEB calibration is performed on a scan-by-scan basis. Among all 490 detectors, there are 44 noisy detectors (30 from prelaunch) and no inoperable detectors in Terra MODIS and 6 noisy detectors (2 from pre-launch) and 13 inoperable detectors (10 from pre-launch) in Aqua MODIS. Most Terra MODIS on-orbit noisy detectors are located on the LWIR FPA and most Aqua inoperable detectors are in band 6 (1.64 $\mu \mathrm{m})$. 


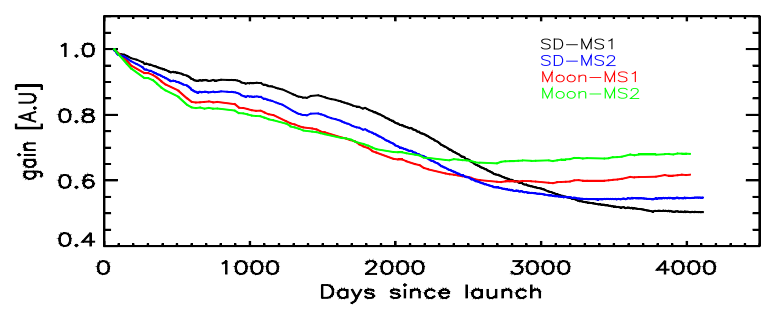

Figure 8 Changes in Terra MODIS band $8(0.41 \mu \mathrm{m})$ gains

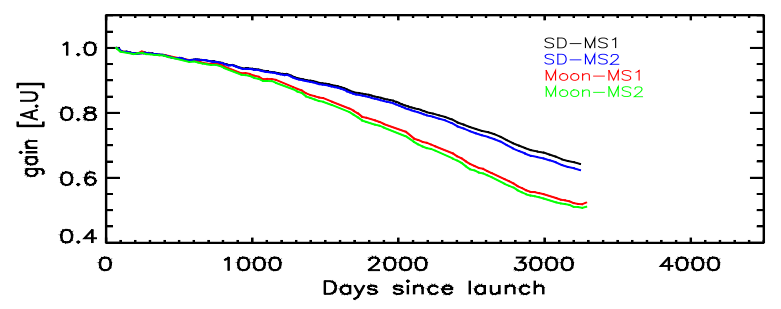

Figure 9 Changes in Aqua MODIS band $8(0.41 \mu \mathrm{m})$ gains

Both spatially and spectrally, Terra and Aqua MODIS have demonstrated excellent stability. Except for a couple of bands, the changes in center wavelengths (CW) and bandwidths (BW) have been less than $0.5 \mathrm{~nm}$ and 1.0 $\mathrm{nm}$, respectively, for most VIS and NIR bands. On-orbit results also show that both Terra and Aqua MODIS have experienced little or no change in their along-scan and along-track band-to-band registration (BBR). For Terra MODIS, most band pairs continue to meet the specifications of $\pm 0.1 \mathrm{~km}$. For Aqua MODIS, large BBR offsets (0.3-0.4 $\mathrm{km}$ in both directions) identified pre-launch remains unchanged on-orbit. It is anticipated that, with dedicated calibration efforts, both Terra and Aqua MODIS will continue to provide quality data products and make great contributions to a broad range of scientific studies and applications.

As both instruments continue to operate beyond their 6-year "design life”, the frequencies of some calibration activities, such as SD and SDSM operations, have been gradually reduced without impacting the existing calibration and data product quality. A newly developed approach of accurate mapping of the SDSM screen transmission has been applied to further improve the accuracy of tracking SD on-orbit degradation. Continuous studies will be conducted to examine the calibration impact, especially on the nonlinear calibration coefficients, due to Aqua cold FPA temperature variations. For the RSB, the most challenging task remains to be accurate determination of changes of sensor response versus scan angle. In addition to OBC and lunar observations at limited scan angles, the use of longterm trends from ground targets over a wide range of scan angles has become an essential part of RVS characterization. As expected, special considerations and efforts have been made in support of science investigations as part of the efforts for collection 6 data product quality assurance.
Many lessons have been learned from both Terra and MODIS instrument design, pre-launch calibration and characterization, on-orbit operation, calibration, and sensor performance, algorithm implementation, LUT updates, and calibration uncertainty assessment. As illustrated in this paper, no two sensors are completely identical and all sensor responses change on-orbit, large or small. Consequently, it is critical to maintain continuous calibration efforts throughout the sensor's entire mission. MODIS lessons have already provided and will continue to provide valuable information for other mission and sensor development, their operation and important calibration activities.

\section{SUMMARY}

Terra and Aqua MODIS have successfully operated for more than 11 and 9 years, respectively. On-orbit calibration performance shows that both instruments and their on-board calibrators continue to be able to provide key design functions and to allow high quality data products to be generated for scientific studies and applications. Except for a couple of problems identified pre-launch, Aqua MODIS on-orbit overall performance has been better and more stable than Terra MODIS, especially for the bands at shorter wavelengths. MODIS experience and lessons learned through all phases will continue to benefit future earthobserving sensors' design, development, pre-launch and onorbit calibration.

\section{REFERENCES}

[1] V. Salomonson, W. Barnes, X. Xiong, S. Kempler, and E. Masuoka, "An Overview of the Earth Observing System MODIS Instrument and Associated Data Systems Performance," Proc. IEEE International Geoscience and Remote Sensing Symposium, doi:10.1109/IGARSS.2002.01025746, pp. 970-972, 2002

[2] X. Xiong, K. Chiang, J. Esposito, B. Guenther, and W.L. Barnes, "MODIS On-orbit Calibration and Characterization,” Metrologia 40, 89-92, 2003

[3] Barnes W.L., X. Xiong, T. Salerno, B. Breen, and C. Salo, "Operational Activities and On-orbit Performance of Terra MODIS On-board Calibrators”, Proceedings of SPIE - Earth Observing Systems X, Vol. 5882, 2005

[4] X. Xiong, K. Chiang, J. Sun, W. Barnes, B. Guenther, and V. Salomonson, "NASA EOS Terra and Aqua MODIS On-orbit Performance,” Adv. Space Res., 43, 413-422, doi:10.1016/j.asr.2008.04.008, 2008

[5] X. Xiong, B.N. Wenny, and W.L. Barnes, "Overview of NASA Earth Observing Systems Terra and Aqua Moderate Resolution Imaging Spectroradiometer Instrument Calibration Algorithms and On-orbit Performance,” J. Appl. Remote Sens., Vol. 3, 032501, doi:10.1117/1.3180864, 2009 